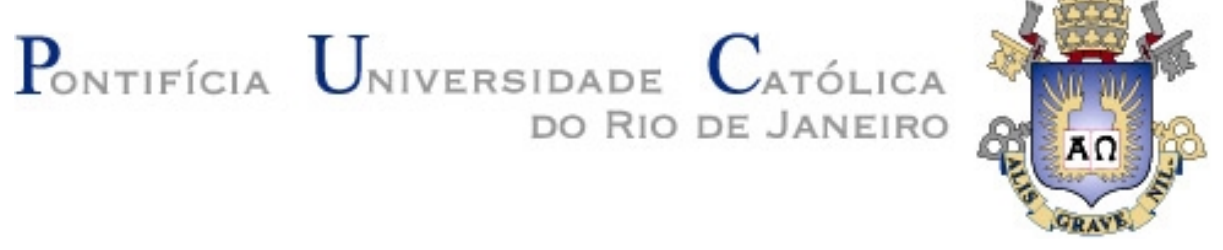

Rodrigo Jurema de Assis Corrêa

\title{
Análise de Desempenho de Enlaces com Modulação Adaptativa Aplicada a Sistemas WCDMA/HSDPA
}

\author{
Dissertação de Mestrado
}

Dissertação apresentada como requisito parcial para obtenção do título de Mestre pelo Programa de PósGraduação em Engenharia Elétrica da PUC-Rio.

Orientador: João Célio Barros Brandão

Rio de Janeiro, julho de 2003 


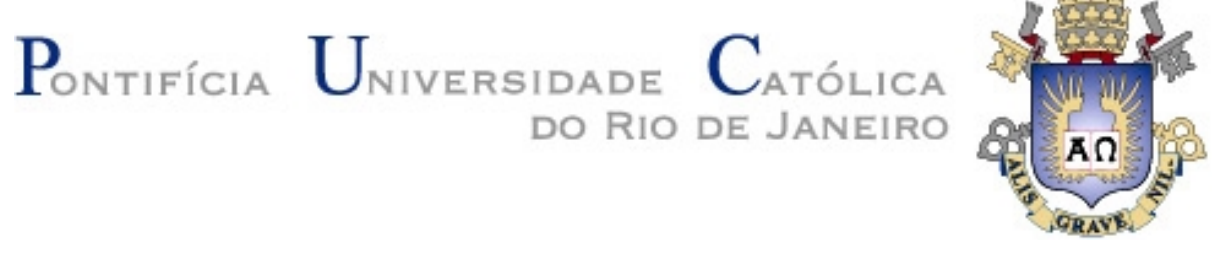

Rodrigo Jurema de Assis Corrêa

\begin{abstract}
Análise de Desempenho de Enlaces com Modulação Adaptativa Aplicada a Sistemas WCDMA/HSDPA
\end{abstract}

Dissertação apresentada como requisito parcial para obtenção do título de Mestre pelo Programa de PósGraduação em Engenharia Elétrica da PUC-Rio. Aprovada pela Comissão Examinadora abaixo assinada.

Prof. João Célio Barros Brandão Orientador CETUC / PUC - Rio

Prof. José Roberto Boisson de Marca CETUC / PUC - Rio

Prof. Marco Antonio Grivet Mattoso Maia CETUC / PUC - Rio

André Noll Barreto ATL

Prof. Ney Augusto Dumont Coordenador(a) Setorial do Centro Técnico Científico - PUC-Rio 
Todos os direitos reservados. É proibida a reprodução total ou parcial do trabalho sem autorização da universidade, da autora e do orientador.

\section{Rodrigo Jurema de Assis Corrêa}

Graduou-se em Engenharia Eletrônica na Universidade Federal de Pernambuco em julho de 2001. Em agosto do mesmo ano iniciou no Centro de Estudos de Telecomunicações da Pontifícia Universidade Católica do Rio de Janeiro seu mestrado na área de sistemas de comunicações móveis celulares.

Ficha Catalográfica

Corrêa, Rodrigo Jurema de Assis
Análise de desempenho de enlaces com
modulação adaptativa aplicada a sistemas
WCDMA/HSDPA / Rodrigo Jurema de Assis Corrêa ;
orientador: João Célio Barros Brandão. - Rio de Janeiro :
PUC-Rio, Departamento de Engenharia Elétrica, 2003.
102 f. : il. ; 30 cm
Dissertação (mestrado) - Pontifícia Universidade
Católica do Rio de Janeiro, Departamento de Engenharia
Elétrica.
Inclui referências bibliográficas
1. Engenharia elétrica - Teses. 2. Telefonia
celular. 3. Modulação digital. 4. WCDMA. 5. HSDPA. 6.
UMTS. I. Brandão, João Célio Barros. II. Pontifícia
Universidade Católica do Rio de Janeiro. Departamento de
Engenharia Elétrica.

CDD: 621.3 
Dedico este trabalho aos meus pais, Plínio Jr. e Silvia, e aos meus irmãos, Plínio Neto e Rafael, por todo amor e incentivo dados durante toda minha vida. 


\section{Agradecimentos}

Ao meu orientador, Professor João Célio Barros Brandão, pela excelente orientação, competência, paciência, confiança e por ter sido um grande amigo. Muito obrigado por tudo!

Ao Professor Marco Antonio Grivet M. Maia, por ter fornecido a estrutura do Laboratório de Redes do CETUC.

À CNPq pela ajuda financeira dada durante o curso, sem a qual seria impossível a realização deste trabalho.

Ao meu amigo e enorme colaborador Robson Domingos Vieira, por toda ajuda na implementação do simulador e pelas excelentes dicas e discussões realizadas.

Aos professores do grupo de sistemas de comunicação do CETUC, pela excelente receptividade e por contribuírem bastante para o meu crescimento profissional.

Aos professores da UFPE, por terem me formado e me preparado para enfrentar este curso.

Aos meus avós, Merval, Maidy, Plínio (in memorian) e Adail, pelo constante apoio, amor e carinho.

Ao meu amigo Abílio Jr. e família, por todo suporte, amizade e companheirismo dados durante o curso.

Ao meu primo Guilherme Jurema e família, por terem sido minha família no Rio de Janeiro.

Ao meu amigo Rui Fibiger e família, por toda ajuda dada no início e durante o curso.

Aos meus tios, tias, primos e primas, pelo eterno incentivo.

A todos os meus amigos de Recife, do Rio de Janeiro e de toda parte do mundo, pelo constante apoio. Valeu! Sem vocês eu não conseguiria!

Aos funcionários da PUC, em especial aos do CETUC, por todo auxílio dado ao longo do curso. 


\section{Resumo}

Jurema de Assis Corrêa, Rodrigo. Análise de Desempenho de Modulação Adaptativa com utilização em sistemas WCDMA/HSDPA. Rio de Janeiro, 2003. xxxp. Dissertação de Mestrado - Departamento de Engenharia Elétrica, Pontifícia Universidade Católica do Rio de Janeiro.

Este trabalho é um estudo sobre o desempenho de um enlace típico do padrão UMTS/WCDMA para sistemas celulares de terceira geração, utilizando a técnica denominada modulação adaptativa. Esta técnica consiste em escolher, para cada quadro de bits transmitido, a modulação mais adequada às condições do canal. Os aspectos básicos do problema foram formulados analiticamente considerando a transmissão com modulações PSK e QAM coerentes em um canal com desvanecimento plano e efeito Doppler. Para considerar a aplicação a um modelo mais realista, foi desenvolvido um programa de simulação capaz de modelar esse mesmo problema considerando os principais componentes do sistema HSDPA (High Speed Data Packet Access), um sub-sistema do UMTS/WCDMA padronizado para transmissão de dados em alta velocidade no enlace de descida. $\mathrm{O}$ desempenho do enlace foi avaliado para diversas situações através da taxa de erro de bit, da taxa de transmissão média e da vazão obtida na transmissão, procurando-se investigar a influência de parâmetros do sistema nesse desempenho.

\section{Palavras-chave}

WCDMA, HSDPA, UMTS, RSR, Modulação Adaptativa 


\section{Abstract}

Jurema de Assis Corrêa, Rodrigo. Performance Analysis of Links with Adaptive Modulation applied to WCDMA/HSDPA Systems. Rio de Janeiro, 2003. xxxp. Dissertação de Mestrado - Departamento de Engenharia Elétrica, Pontifícia Universidade Católica do Rio de Janeiro.

This work is a study about the performance of a typical UMTS/WCDMA link for third generation mobile communications systems which uses the adaptive modulation technique. This technique consists of choosing, for each transmitted frame, the most efficient modulation according to the channel conditions. The basic aspects of the problem were analytically examined considering transmissions with PSK and QAM coherent techniques in a flat fading channel with Doppler effect. A simulator was developed in order to consider the application in a more realistic model. This simulator was capable of modeling this problem considering the main aspects of the HSDPA (High Speed Data Packet Access) system, which is a subsystem of UMTS/WCDMA that is standardized for high-speed data transmission in the downlink. The link level performance was evaluated for many different situations through average transmission bit error rate and throughput, investigating the influence of the system parameters in this performance.

\section{Key Words}

WCDMA, HSDPA, UMTS, SNR, Adaptive Modulation 


\section{Sumário}

1 Introdução

2 WCDMA

2.1. Principais Características 18

2.2. Arquitetura do WCDMA 20

2.3. Camada Física do WCDMA 22

2.3.1. Estrutura dos Canais Físicos 22

2.3.2. Modulação e Espalhamento no Enlace de Subida 23

2.3.3. Modulação e Espalhamento no Enlace de Descida 27

2.3.4. Estrutura do Quadro no Enlace de Subida 29

2.3.5. Estrutura do Quadro no Enlace de Descida 31

2.3.6. Codificação do Canal 33

3 Modulação e Codificação Adaptativa 34

3.1. Técnicas de Adaptação de Enlace 35

3.1.1. Retransmissão Automática Híbrida (H-ARQ) 37

3.1.2. Sistemas MIMO 38

3.1.3. Sistemas Multi-portadoras $\quad 39$

3.2. Modulação e Codificação Adaptativa 41

3.2.1. Análise de Desempenho 42

3.2.2. Critérios de Adaptação de Enlace 44

3.2.2.1. Adaptação Baseada no RSR Médio 45

3.2.2.2. Adaptação Baseada em Estatísticas Múltiplas do RSR 46

4 HSDPA $\quad 47$

4.1. Introdução 47

4.2. Impactos do HSDPA na Arquitetura da Rede 49

4.3. Camada Física do HSDPA 51

4.3.1. HS-PDSCH 51

4.3.2. HS-PSCCH 54 
5 Análise de Desempenho $\quad 57$

5.1. Modelo do Sistema de Transmissão Digital 57

5.2. Descrição do Simulador 64

5.3. Parâmetros de Desempenho 67

5.3.1. Razão Sinal-Ruído-Interferência, na Ausência de Desvanecimento67

5.3.2. $E_{b} / N_{0}$ na Ausência de Desvanecimento 67

5.3.3. $E_{b} / N_{0}$ "Instantânea" 68

5.3.4. $E_{b} / N_{0}$ Média 68

5.3.5. Taxa de Erro de Bit e Taxa de Transmissão Médias 69

5.3.6. Vazão 70

5.4. Resultados 72

5.4.1. Validação do Simulador 72

5.4.2. Taxa Média de Transmissão e Taxa de Erro Média 78

5.4.3. Desempenho na transmissão de pacotes 80

5.4.4. Impacto de alguns aspectos da implementação 84

5.4.5. Adaptação a partir do Sinal Recebido 87

6 Conclusão 92

Apêndice A - Eficiência Espectral de Sistemas com e sem Potência Adaptativa 95

Apêndice B - Probabilidade de Erro em um Canal com Desvanecimento Rayleigh

Apêndice C - Lista de Acrônimos 101

$\begin{array}{ll}\text { Referências Bibliográficas } & 102\end{array}$ 


\section{Lista de figuras}

Figura 1: Arquitetura do UMTS/WCDMA. 20

Figura 2: Transmissão paralela dos DPDCH/DPCCH com a presença/ausência de dados.

Figura 3: Relação entre espalhamento e scrambling.

Figura 4: Modulação e espalhamento no enlace de subida. 25

Figura 5: Modulação e espalhamento no enlace de descida. 27

Figura 6: Exemplo de árvore de códigos de um PDSCH. 29

Figura 7: Estrutura de quadro do DPDCH/DPCCH no enlace de subida. $\quad 30$

Figura 8: Estrutura de quadro do DPDCH/DPCCH no enlace de descida. $\quad 32$

Figura 9: Modelo de um sistema de comunicação digital. 35

Figura 10: $\mathrm{BER} \times \mathrm{E}_{\mathrm{b}} / \mathrm{N}_{0}$ com e sem codificação. 36

Figura 11: Diagrama de um sistema de transmissão MIMO. 39

Figura 12: Eficiência espectral para várias modulações diferentes em função de RSR. 43

Figura 13: Diagrama de fluxo básico de um sistema adaptativo. 44

Figura 14: Arquitetura do WCDMA antiga e com a modificação do HSDPA. 49

Figura 15: Códigos alocados para o HS-PDSCH. 51

Figura 16: Compartilhamento do HS-PDSCH por multiplexação de códigos e de tempo. 52

Figura 17: Relação de tempo entre os canais HS-PDSCH e HS-PSCCH. 55

Figura 18: Modelo de um sistema de transmissão digital no enlace de descida com adaptação de enlace. $\quad 58$

Figura 19: Espectro de potência Doppler de um desvanecimento Rayleigh. $\quad 62$

Figura 20: Receptor do usuário $i$.

Figura 21: Curva do $B E R \times E_{b} / N_{0}$ para obtenção dos limiares de adaptação para

$$
B E R_{\text {req }}=10^{-2} .
$$

Figura 22: Desvanecimento do canal móvel em função do tempo. 72

Figura 23: Função de Distribuição Cumulativa da envoltória do sinal com desvanecimento de Rayleigh. 
Figura 24: Variação da amplitude do sinal em um quadro, para 3 km/h. $\quad 73$

Figura 25: Variação da amplitude do sinal em um quadro, para $50 \mathrm{~km} / \mathrm{h} . \quad 74$

Figura 26: Variação da amplitude do sinal em um quadro, para 120 km/h. $\quad 74$

Figura 27: Comparação das probabilidades de erro para o QPSK. 75

Figura 28: Comparação das probabilidades de erro para o 8-PSK. 76

Figura 29: Comparação das probabilidades de erro para o 16-QAM. 76

Figura 30: Comparação das probabilidades de erro para o 64-QAM. 77

Figura 31: Taxa de Transmissão Média X SINR para $B E R_{\text {req }}=10^{-2} \mathrm{e}$

$$
\begin{array}{ll}
T_{\text {quadro }}=3 \times T_{\text {slot }} . & 78
\end{array}
$$

Figura 32: $\overline{B E R} \times$ SINR para $B E R_{\text {req }}=10^{-2}$ e $T_{\text {quadro }}=3 \times T_{\text {slot }} . \quad 79$

Figura 33: Freqüência das modulações X SNR para 3 km/h. 79

Figura 34: Vazão X SINR para $B E R_{\text {req }}=10^{-2}$ e $T_{\text {quadro }}=3 \times T_{\text {slot }} . \quad 80$

Figura 35: $\overline{P E R}$ X SINR para $B E R_{\text {req }}=10^{-2}$ e $T_{\text {quadro }}=3 \times T_{\text {slot }} . \quad 81$

Figura 36: Vazão teórica com modelagem por pacotes. 82

Figura 37: Comparação entre as vazões para $3 \mathrm{~km} / \mathrm{h}$, com critérios diferentes para determinação dos limiares.

Figura 38: Comparação entre as vazões para $120 \mathrm{~km} / \mathrm{h}$, com critérios diferentes para determinação dos limiares. 83

Figura 39: Vazão X SINR com limiares otimizados e $T_{\text {quadro }}=3 \times T_{\text {slot }}$. $\quad 84$

Figura 40: Efeito do atraso na realimentação do sistema para $3 \mathrm{~km} / \mathrm{h}$. 85

Figura 41: Efeito do atraso na realimentação do sistema para $120 \mathrm{~km} / \mathrm{h} . \quad 85$

Figura 42: Vazão X SINR para $3 \mathrm{~km} / \mathrm{h}$. 86

Figura 43: Comparação entre as vazões para $120 \mathrm{~km} / \mathrm{h}$, utilizando técnicas de estimação do canal diferentes. $\quad 87$

Figura 44: Diagrama de blocos do receptor. $\quad 88$

Figura 45: Comparação entre métodos de adaptação diferentes para 3 km/h. $\quad 90$

Figura 46: Comparação entre métodos de adaptação diferentes para 120 km/h. 91 


\section{Lista de tabelas}

Tabela 1: Taxas de dados do DPDCH no enlace de subida. 31

Tabela 2: Taxas de dados do DPDCH no enlace de descida. 33

Tabela 3: Taxas de bit máximas para 15 códigos paralelos. 53

Tabela 4: Comparação entre HS-PDSCH e PDSCH. 54

Tabela 5: Limiares de adaptação para $B E R_{\text {req }}=10^{-2}$. 66

Tabela 6: Limiares de adaptação e taxas de transmissão para as diferentes $\begin{array}{ll}\text { modulações. } & 70\end{array}$

Tabela 7: Principais parâmetros do simulador do enlace de descida do WCDMA/HSDPA.

Tabela 8: Limiares de adaptação para a modelagem que maximiza a vazão. $\quad 82$

Tabela 9: Valores de A e B para diferentes modulações. 97

$\begin{array}{ll}\text { Tabela 10: Principais abreviaturas. } & 101\end{array}$ 